\title{
Study of Thermal Properties of Granite Powder (Scrap) Reinforced Polyester Resin Composite
}

Shujit Chandra Paul*, Muhammed Yusuf Miah, Abdul Gafur and Rajib Chandra Das

Glass and Ceramics Engineering Department, Bangladesh University and Engineering Technology, Dhaka, Bangladesh

\begin{abstract}
Composite materials are now widely used in engineering application and the scope of its application is widespread mainly for its contrapuntal properties. In this research work composites were prepared by crosslinking the polyester resin with Methyl Ethyl Ketone Peroxide and finally reinforced with the Granite scrap powder. Granite stone as filler was used at $20 \%, 30 \%, 40 \%$ and $50 \%$ with respect to the weight of unsaturated polyester resin with styrene monomer at $7.5 \%$ as constant basis mentioning that $0 \%$ sample (without Granite Filler) was also prepared to compare the results with it. Thermal analysis of the prepared composite such as Thermo Gravimetric Analysis (TGA), Thermo-mechanical Analysis (TMA) and Thermal Conductivity of the composites were analyzed along with the SEM analysis to find out correlation between thermal properties with composite surface topology. Thermo gravimetric analysis is done to determine the kinetics of thermal degradation and Thermo mechanical analysis is to determine expansion co-efficient along with $\mathrm{Tg}$ of polyester resin and granite blends composite. To avoid the thermo-oxidative degradation, thermal analysis was carried out for samples in nitrogen atmosphere at heating rates of $20^{\circ} \mathrm{C} / \mathrm{min}$. Thermal Conductivity analysis is performed to determine the ability of the composite to conduct heat at various temperatures.
\end{abstract}

Keywords: Composite; TGA; TMA; Thermal conductivity; Coefficient of thermal expansions; Tg; SEM

\section{Introduction}

Granite powder is a byproduct of granite stones obtained during the sizing of granite slabs and has no end use. It is composed mainly of quartz and feldspar with minor amounts of mica, amphiboles and other minerals [1]. Granite a non-biodegradable material- poses numerous hazards if the stone waste is dumped on land or when Fine particles get mixed with air. So such waste material needs to be utilized meaningfully in an economic way. At the present time, development of some polymer based composites with industrial waste materials (with no end use) as fillers / reinforcements has gained the popularity [1-3]. But so far little development has been reported on the utilization of waste granite powder as the filler in thermosets. Hence if such granite powder can be used as the filler, it would be beneficial in reducing the overall cost of the composites and it would be useful for proper disposal of granite powder [4].

Unsaturated polyester resins usually has the special properties like; it is easy to process and comparatively a competitive price, which makes the resin more usable and reliable for fabrication of polymer composite [5]. Beside this some other applied properties of this resin such as; high corrosion resistance, good mechanical property and the most desirable light weight properties make it usable in different area including automotive filed, construction, and electrical application and coating [6-8].

As Polyester resin based composites are usually heat sensitive therefore their thermal analysis is very important to find out the composite thermal stability, optimum temperature of usability, expansion parameter along with other related thermodynamic properties [9-12].

Polyester based composites are heat sensitive and mainly initiate the degradation due to thermal changes. All types of degradation of polyester composites is initiated by the breakdown of highly strain portion of polystyrene cross-links forming the reactive free radicals that promotes further decomposition of cross-linked structure [1315]. Polyester decomposition is arguably a two stage process, single stage Arrhenius kinetics is sufficiently accurate to model the process [16]. During the thermal degradation process ester group exchange of polyester structure and the $\beta$ - $\mathrm{CH}$ hydrogen transfer among intermolecules are prominently responsible at the initial stage of thermal degradation $[17,18]$.

\section{Experimental}

\section{Raw material and composites preparation}

Stones (Scraps) collected from local markets contain various mud and debris. These scraps are cleaned with water and then dried under sun. Grinding is done in a high speed mortar mill for 10 minutes for every 50-60 gm. of sample. Sieve analysis of grinded powder is done to separate various particle sizes to ensure that we can use comparatively the lower particle size powder mentioning that the lower particle size ensures better mechanical properties. Grinded powder is dried in oven for 24 hours at $60^{\circ} \mathrm{C}$. Polymerization (Free-radical Polymerization) is usually initiated by Methyl Ethyl Ketone Peroxide (MEKP) with Unsaturated Polyester Resin and finally the crosslinking properties are increased by styrene monomer which also ensures the termination of reaction.

During stirring granite powder is added and finally the mixture is poured into the three different shaped of open molds. The molded melt is then kept for 24 hours for curing.

${ }^{*}$ Corresponding author: Shujit Chandra Paul, Glass and Ceramics Engineering Department, Bangladesh University and Engineering Technology, Dhaka, Bangladesh, Tel: +8801515216790; E-mail: shujitpaul@yahoo.com

Received August 02, 2016; Accepted September 17, 2016; Published October 19, 2017

Citation: Paul SC, Miah MY, Gafur A, Das RC (2017) Study of Thermal Properties of Granite Powder (Scrap) Reinforced Polyester Resin Composite. J Adv Chem Eng 7: 159. doi: 10.4172/2090-4568.1000159

Copyright: (C) 2017 Paul SC, et al. This is an open-access article distributed under the terms of the Creative Commons Attribution License, which permits unrestricted use, distribution, and reproduction in any medium, provided the original author and source are credited. 


\section{Thermo-gravimetric analysis}

The thermo-gravimetric analysis was performed on a TGA / DTA Machine (TG/DTA 6300) which instrument is capable of performing Thermogravimetric (TG), Differential thermal analysis (DTA) and Differential Thermogravimetric (DTG) at the same time. To avoid any kinds of oxidative degradation the TGA/DTA analysis were conducted under a constant flowing atmosphere of nitrogen (Inert atmosphere) at a purge rate of $200 \mathrm{ml} / \mathrm{min}$. About $3.5 \pm 0.3 \mathrm{mg}$ of sample was taken from the prepared composite by means of a blade and the size of the collected sample was small enough for a smooth degradation under the provided heating condition of TGA/DTA machine (Table 1). The sample was then placed in an alumina sample pan. TGA/DTA machine has sample balance beam and reference balance beam which are independently supported by driving coil/pivot. A change in weight at the beam end is transferred to the opposite end of the beam via the driving coil/pivot and the optical position sensors detect changes in the position of a slit and finally converted into weight signal. Alumina $\left(\mathrm{Al}_{2} \mathrm{O}_{3}\right)$ is used as a reference material.

\section{Thermo mechanical analysis}

TMA is a reliable instrument for determination of various thermal properties of composite materials involving the determination of dimension change of the sample as a function of temperature, time, or force. Such type of analyzer is mainly used for determining the thermal expansion coefficients, beside this it can also be used to determine glass transition temperature. A sample heating/Cooling rate of $5^{\circ} \mathrm{C} / \mathrm{min}$ was used to determine the thermal expansion coefficient along with the $\mathrm{Tg}$ values between the temperature interval of $30^{\circ} \mathrm{C}-120^{\circ} \mathrm{C}$. Coefficient of linear thermal expansion was calculated above and below the $\mathrm{Tg}$ along with the entire temperature interval The coefficient of thermal expansion $(\alpha)$ is determined using the equation [19].

$$
\alpha=\frac{\Delta \mathrm{L}}{\mathrm{L} \Delta \mathrm{T}}
$$

Where, $\mathrm{L}=$ Initial Length of sample

\section{Thermal conductivity}

Thermal conductivity- the ability to conduct heat of a solid body, is mainly depends on the gradient of temperatures between the two medium. When two solid mediums are maintained in a temperature gradient state heat is usually transferred from the higher temperature medium to low temperature medium. The value of thermal conductivity can be calculated from the following equation:

$$
\mathrm{K}=\frac{\mathrm{mc}\left(\frac{\mathrm{dT}}{\mathrm{dt}}\right) \mathrm{x}}{\mathrm{A}(\mathrm{T} 1-\mathrm{T} 2)}
$$

Where, $\mathrm{K}=$ Coefficient of thermal conductivity of the metallic disc, $\mathrm{A}=$ Area of the sample in contact with the metallic disc, $\mathrm{x}=$ Thickness of the sample, $m=$ Mass of the metallic disc, $c=$ The specific heat of the metallic disc, $\mathrm{dT} / \mathrm{dt}=$ Rate of cooling of the metallic disc at T1 (Slope of Temperature vs. Time Curve).

Thermal conductivity is determined by ASTM E1530 method where disc shaped specimens with a diameter of $50 \mathrm{~mm}$ and thickness of $10 \mathrm{~mm}$ is reacquired to determine the thermal conductivity values of granite filled composite. A known constant heat with the help of an oil bath is applied from one side of the specimen until a thermal equilibrium is attained between two discs and after achieving the steady state condition the temperature of the top and bottom surfaces were measured by using thermocouples installed on both surfaces of the specimen [20].

\section{Results and Discussion}

\section{Thermo-gravimetric analysis}

The thermo-gravimetric (TG) and derivative thermo-gravimetric (DTG) analysis for polyester resin composite is presented in Figure 3. The topmost lines represent the \%TG which indicates the steps of degradation, the middle lines represent DTA from which exothermic or endothermic information is obtained and the bottom group of line represents the DTG which usually gives the maximum degradation rate along with the temperature for maximum degradation.

As granite powder mainly consists of various crystalline components therefore the first stages of degradation (up to about $300^{\circ} \mathrm{C}$ ) represent the evaporation of the hydrated water of crystal components. TG graph shows that all composite samples degraded by single step degradation process. The degradation mainly occurs due to the random scission of cross-linked structure as well as branching of polymer chain.

It is seen that the onset temperature shifts to higher temperature with the addition of filler which is an indication of increasing thermal stability of composite with successive addition of filler content. Again

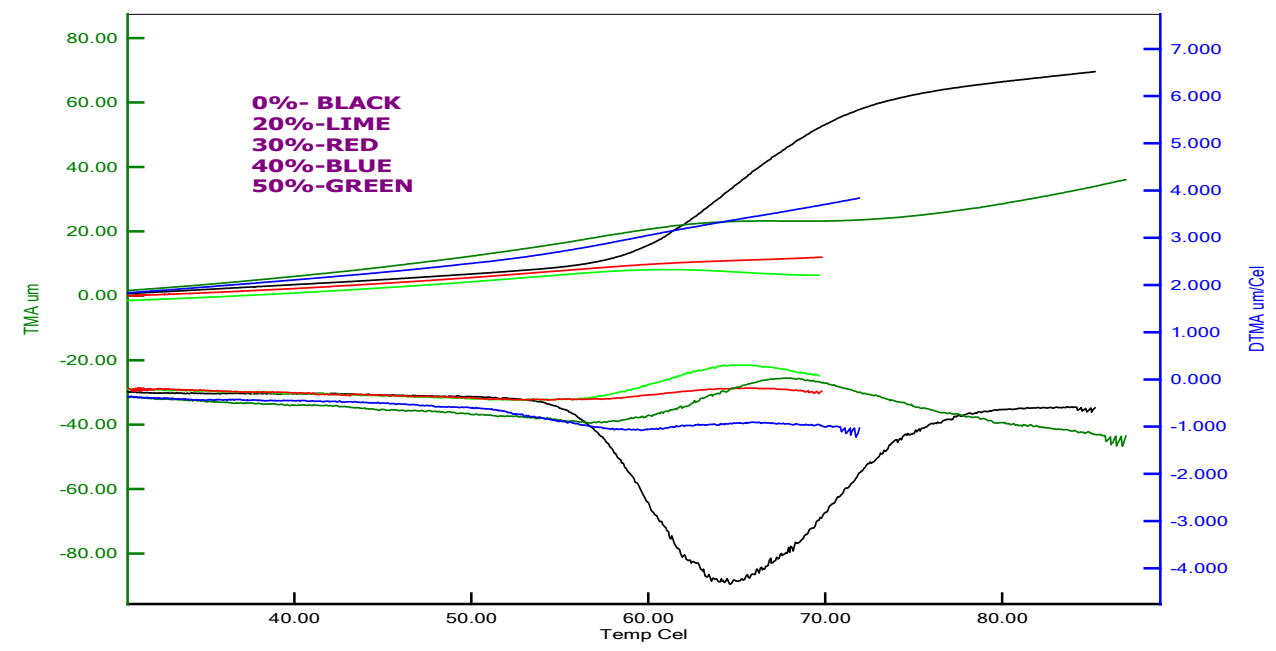

Figure 1: Thermo mechanical Analysis of Granite filled composite. 




Figure 2: Thermo-gravimetric analysis of Granite filled composite.



Figure 3: Thermal Conductivity of Granite filled Composite.

the DTG value indicated that the rate of degradation during thermal decomposition decreases with the increase in filler content in composite materials. Due to this thermal stability about $65 \%$ un-decomposed residue remains for SG-50\% Sample. From the DTG data we see that the maximum degradation rate is obtained for $0 \%$ filled composite whereas the degradation rate falls gradually with the increasing of filler content.

\section{Thermo-mechanical analysis}

The top most line indicates TMA line from which values of $\alpha$ is calculated calculating the slope of the line. It is seen that the $0 \%$ Sample has the highest slope and hence highest $\alpha$ value. The bottom group of line represents the DTMA lines from which the maximum expansion rate as well as the temperature for maximum expansion is obtained.

The fall of $\mathrm{Tg}$ in Granite-polyester composites can be attributed to two factors: the incomplete curing reaction and the increase in the free volume of the system. The decrease in the free volume is proposed to be responsible for the increased Tg values because of the addition of granite filler. In addition, the increase in $\mathrm{Tg}$, may be attributed to the nano-reinforcement effect and the segmental level restriction of the motion by the incorporation of granite powder into the polyester matrix leads to increase in $\mathrm{Tg}$ [21-23].

From the experimental results it is observed that increase the addition of filler to composites reduces the thermal expansion coefficient. It has been noticed that $50 \%$ Volume filled composite exhibited less thermal expansion coefficient both below and above the $\mathrm{Tg}$ and hence through the entire range of temperature interval of test. Actually the interaction between polymer with the filler increase with the filler content and this good filler- matrix interaction is responsible for the lower thermal expansion coefficient value which is also a good indication of stability of composite in a heated environment. The similar results (Table 2) are also obtained by many studies [24].

\section{Thermal conductivity}

The thermal conductivity values obtained from the experimental study for the particulate filled polyester composites with varied proportion of Granite Filler is shown in following Figures (Figure 1 and 2). From the experiment it is seen that the value thermal conductivity 
Citation: Paul SC, Miah MY, Gafur A, Das RC (2017) Study of Thermal Properties of Granite Powder (Scrap) Reinforced Polyester Resin Composite. J Adv Chem Eng 7: 159. doi: 10.4172/2090-4568.1000159

Page 4 of 5

\begin{tabular}{|c|c|c|c|c|c|}
\hline Sample & $\mathrm{T}_{\text {onset, }}{ }^{\circ} \mathrm{C}$ & $\mathbf{T}_{\text {endset, }}{ }^{\circ} \mathrm{C}$ & $\mathrm{T}_{\text {ma x imun, }}{ }^{\circ} \mathrm{C}$ & DTG, mg/min & $\begin{array}{l}\text { Residue, \% } \\
\text { (At } 500^{\circ} \mathrm{C} \text { ) }\end{array}$ \\
\hline $0 \%$ Sample (No Filler) & 329.5 & 416.6 & 386.9 & 1.148 & 5.2 \\
\hline SG-20\% & 350.5 & 414.8 & 388.7 & 0.811 & 49.8 \\
\hline SG-30\% & 351.0 & 405.6 & 378.9 & 0.647 & 50.0 \\
\hline SG-40\% & 355.9 & 410.5 & 383.6 & 0.377 & 56.4 \\
\hline SG-50\% & 363.3 & 407.8 & 379.6 & 0.345 & 64.9 \\
\hline
\end{tabular}

Table 1: Summary of TG/DTA analysis.

\begin{tabular}{|c|c|c|c|c|c|}
\hline Sample & $\mathrm{Tg},{ }^{\circ} \mathrm{C}$ & $\begin{array}{c}\alpha, \text { Below Tg } \\
1 /{ }^{\circ} \mathrm{C}\end{array}$ & $\begin{array}{c}\alpha, \text { After Tg } \\
1 /{ }^{\circ} \mathrm{C}\end{array}$ & $\begin{array}{c}\alpha, \text { Entire Temperature Interval } \\
1 /{ }^{\circ} \mathrm{C}\end{array}$ & $\begin{array}{l}\text { DTMA } \\
\mu \mathrm{m} /{ }^{\circ} \mathrm{C}\end{array}$ \\
\hline $\begin{array}{l}0 \% \text { Sample } \\
\text { (No filler) }\end{array}$ & 59 & $1.42 \times 10^{-4}$ & $1.20 \times 10^{-3}$ & $7.27 \times 10^{-5}$ & 4.33 \\
\hline SG $20 \%$ & 48 & $4.93 \times 10^{-5}$ & $1.01 \times 10^{-4}$ & $7.27 \times 10^{-5}$ & 1.084 \\
\hline SG $30 \%$ & 48 & $6.67 \times 10^{-5}$ & $6.78 \times 10^{-5}$ & $6.69 \times 10^{-5}$ & 0.669 \\
\hline SG $40 \%$ & 53 & $5.77 \times 10^{-5}$ & $3.41 \times 10^{-5}$ & $4.56 \times 10^{-5}$ & 0.432 \\
\hline SG $50 \%$ & 53 & $3.5 \times 10^{-5}$ & $3.6 \times 10^{-5}$ & $3.55 \times 10^{-5}$ & 0.413 \\
\hline
\end{tabular}

Table 2: Summary of TMA analysis.
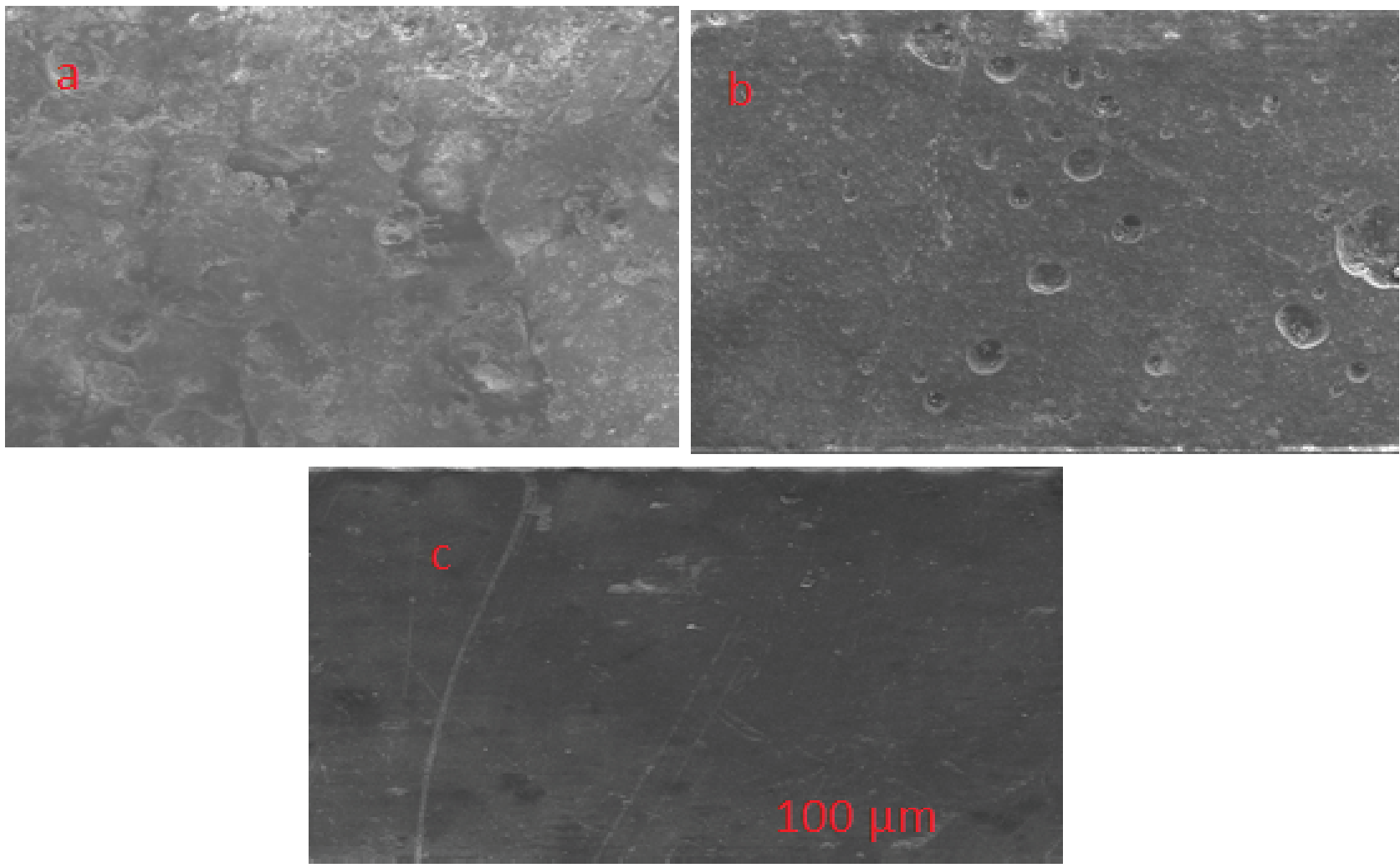

Figure 4: SEM analysis of (a) $20 \%$, (b) $40 \%$ and (c) $50 \%$ Granite filled composite.

is increased with increase of filler contents. Heat is usually transferred by means of vibration of nearest molecules so with the increase of filler contents this conduction becomes easy.

Again with the increase in filler contents the porosity decreases (Figure 4) and hence the ability of the sample to conduct heat is also rises [25]. The thermal conductivity value for $40 \%$ and $50 \%$ sample is almost same because the compactness of both the samples is almost similar (Figure 4). This is due to the fact that the heat usually passed through a solid body by conduction where heat is usually passes through vibration of the nearest molecules.

\section{SEM analysis}

With the increase in filler content the porosity of the composite surfaces decrease and the composite become more compact giving a densely packed structure.

\section{Conclusion}

In this research work thermal decomposition of unsaturated polyester resin based granite composite has been investigated by thermo-gravimetric and thermo-mechanical method along with thermal conductivity analysis. With the increase in addition of filler 
there is a reduction in thermal expansion coefficient as it is seen from the composites filled by (50\% Volume) when compared with $(20 \%$ Volume) filled composites. From the obtained results it is observed that composites filled by (20\% Volume) exhibited low thermal conductivities. Composites filled by ( $50 \%$ Volume) exhibited maximum thermal conductivity $(3 \mathrm{~W} / \mathrm{m} . \mathrm{k})$. The results of this study indicate that the waste granite scrap material reinforced composites possess good thermal insulating and stability properties. Hence, the newly developed composite material could be used for construction materials.

\section{Acknowledgments}

I would like to thank to all Coordinator of "Pilot Plant and Product Development Center, Bangladesh Scientific and Industrial Research Council" for their assistance throughout my works. I am also greatly indebted to Department of "Glass and Ceramics Engineering Department", Bangladesh University and Engineering Technology", for their assistance in SEM analysis without which it was quite difficult to complete my thesis work.

\section{References}

1. Kareem AA (2013) Mechanical properties of granite powder as a filler for polycarbonate toughened epoxy resin. International Journal of Pharma Sciences 3: 254-257.

2. Ramakrishna HV, Padmapriya S, Rai SK (2007) Flexural, compression, chemical resistance and morphology studies on granite powder filled epoxy and acrylonitrile butadiene styrene (ABS) toughened epoxy matrices. Journal of Applied Polymer Science 104: 171-177.

3. Ramakrishna HV, Padmapriya S, Rai SK (2006) Effect on the mechanical properties and water absorption of granite powder composites on toughening epoxy with unsaturated polyester and unsaturated polyester with epoxy resin. Journal of Reinforced Plastics and Composites 25: 17-32.

4. Baskaran R, Sarojadevi M, Vijayakumar CT (2014) Utilization of Granite Powder as Filler for Vinyl Ester Resin. Malaysian Polymer Journal 9: 39-44.

5. Atta AM, Elnagdy SI, Raouf A (2005) Compressive Properties and Curing Behaviour of Unsaturated Polyester Resins in the Presence of Vinyl Ester Resins Derived from Recycled Poly(ethylene terephthalate). J Polym Res 12: 373-383.

6. Selly J (1988) Encyclopedia of Polymer Science and Engineering. Mark HF (ed.), Willey, New York, USA 12: 256-290.

7. Kramer H (1992) Polyester Resin, Unsaturated, Ullmanns. Encyclopedia of Industrial Chemistry. Weinheim 21: 217.

8. Giovanilton FDS, Fernando LC, Sanchez CG (2009) Influence of the particle size in kinetic of pyrolysis of unsaturated polyester. 20th International Congress of Mechanical Engineering, November 15-20, Gramado, RS, Brazil.

9. Budrugeac P, Segal E, Alice LP (1996) Thermal degradation of a styrenated unsatured polyester resin. Journal of Thermal Analysis 46: 1313-1324.
10. Budrugeac $P$, Peter AL, Segal E (1996) Approximation of the TG curve through a temperature power series in order to evaluate the non-isothermal kinetic parameters of solid-gas decompositions and degradation. Thermochimica Acta 275: 193-213.

11. Budrugeac $P$, Segal $E$ (1997) Thermo oxidative degradation of an unsaturated polyester resin the effect of oxygen pressure on the kinetics. Journal of Thermal Analysis 49: 183-191.

12. Budrugeac $P$ (2001) Polymer Degradation and Stability. 47: 125-131.

13. Gibson AG, Hume J (1995) Fire performance of composite panels for large marine structures. Plastics, Rubber and composites, Processing and Application 23: 175- 183.

14. Arii T, Ichihara S, Nakagawa H, Fujii N (1998) A kinetic study of the thermal decomposition of polyesters by controlled-rate Thermogravimetric Thermochimica Acta 319: 139-149.

15. Mouritz AP, Gibson AG (2006) Fire properties of polymer composites Materials Springer, Netherland, 143: 32-97.

16. Tagle L, Hand FRD (1992) Thermchim Acta 200: 281-291.

17. Pielichowski K, Hamerton I (1998) TGA/FTi. R. studies on the thermal stability of poly (vinyl chloride) blends with a novel colourant and stabilizer: 3-(2, 4-dichlorophenylazo)-9-(2, 3-epoxypropane). J Polym 39: 241-244.

18. Kiran Ciliz N, Ekinci E, Snape CE (2000) Recycling of plastic waste via pyrolysis. Resources, Conservation Recycling 29: 273-283.

19. Nakka JS, Jansen KMB, Ernst LJ (2011) Effect of chain flexibility in the network structure on the viscoelasticity of epoxy thermosets. J Polym Res 18: 18791888.

20. Devendra K, Rangaswamy T (2013) Thermal conductivity and therma expansion coefficient of gFRP composite laminates with fillers. Mech Confab 2: 39-44.

21. Zhang J, Zhang W, Guan D (2016) Preparation and properties of epoxy resin/ polyhedral oligomeric silsesquioxane hybrid materials. Polym Bull 73: 113-123.

22. Miao P, Chunling Z, Liu B, Jianxin M (2013) Dielectric and Thermal Properties of Epoxy Resin Nano composites containing Polyhedral Oligomeric Silsesquioxane. Journal of Materials Science Research 2: 153-162.

23. Yao F, Wu Q, Lei $Y$, Guo W, Xu Y (2008) Thermal decomposition kinetics of natural fibers: Activation energy with dynamic thermogravimetric analysis. Polymer Degradation and Stability 93: 90-98.

24. Devendra K, Rangaswamy T (2012) Evaluation of Thermal Properties of E-Glass/ Epoxy Composites Filled by Different Filler Materials. International Journal of Computational Engineering Research 2: 2250-3005.

25. Amaral P, António C Luís L, Paula R, António P, José CL (2013) On the Use of Thermal Properties for Characterizing Dimension Stones. Key Engineering Materials 548: 231-238. 